\title{
Collective effects in the energy loss of large hydrogen clusters
}

\author{
F. Javier Pérez-Pérez and Isabel Abril \\ Departament de Física Aplicada, Universitat d'Alacant, Apartat 99, E-03080 Alacant, Spain \\ Rafael Garcia-Molina \\ Departamento de Física, Universidad de Murcia, Apartado 4021, E-30080 Murcia, Spain \\ Néstor R. Arista \\ Instituto Balseiro, Centro Atómico Bariloche, RA-8400 Bariloche, Argentina
}

(Received 15 May 1996)

\begin{abstract}
The energy loss of large molecular-hydrogen clusters incident on aluminum and amorphous carbon targets is analyzed as a function of the cluster size and velocity, using a dielectric formalism to describe the electronic interactions between the projectile and the target. The dependence of the energy loss with the cluster size predicts a marked different behavior for aluminum or amorphous carbon. The energy loss of $\mathrm{H}_{2}$ clusters in aluminum indicates that for each cluster velocity there is a "resonant" cluster size for which the intermolecular contribution to the energy loss is maximum. On the other hand, the intermolecular contribution to the energy loss of $\mathrm{H}_{2}$ clusters in amorphous carbon saturates with the cluster size. The origin of this behavior lies in the low-energy plasmon present in the energy loss function of amorphous carbon. We also discuss the consequences of a proper description of the dielectric properties of the target. [S1050-2947(96)08810-5]

PACS number(s): $34.50 . \mathrm{Bw}, 36.40 .-\mathrm{c}$
\end{abstract}

\section{INTRODUCTION}

The interaction of atomic particles with condensed matter has been studied in depth and profusely from the beginning of this century. Nonetheless, during recent years a new interest has grown in the interaction of polyatomic projectiles with matter [1], due to the possibility of producing clusters made by a large number of atoms, and at relatively high kinetic energies, which now extends up to the MeV/atom range.

The interest in cluster beams interacting with solids and plasmas was motivated by the fact that it provides additional information about a variety of atomic-collision phenomena and also due to several applications (like implantation, surface analysis, sputtering, determination of geometrical structures of polyatomic ions, inertial fusion drivers, etc.) [1]. In a pioneering work, Brandt et al. [2] measured and calculated the energy loss of $\mathrm{H}_{2}{ }^{+}$and $\mathrm{H}_{3}{ }^{+}$beams in thin foils and compared the results with the stopping power corresponding to proton beams at the same velocity. The conclusion of this paper was that the energy loss of the cluster shows important differences, called vicinage effects, with respect to the energy loss of its constituents considered separately. The origin of this effect lies in the interferences produced by the electronic excitations of the target due to the correlated motion of the particles that form the cluster.

At present, the study of the basic interactions between fast molecular-ion beams interacting with solids has received renewed interest, both theoretically [3-8] and experimentally [3,9-11]. However, works related to the interaction of large clusters with matter are scarcer, although we should quote recent experimental work concerning the interaction of large hydrogen and carbon clusters with solids [12-16]. The experimental stopping power ratio of hydrogen clusters in car- bon foils and its dependence on both cluster velocity and size was presented in Ref. [15], for cluster energies in the range of keV/amu and cluster sizes up to 25 atoms. At low energies they [15] found that the ratio between the total energy loss of $\mathrm{a} \mathrm{H}_{n}^{+}$cluster and the energy loss of $n$ individual protons at the same velocity is less than unity, but it becomes larger than unity at high energies, in agreement with earlier observations using $\mathrm{H}_{2}{ }^{+}$ions $[17,18]$.

Saturation effects in the cluster energy loss due to the cluster size were observed at least for the lower cluster energies [15]. Recently the stopping power of carbon clusters and fullerene ions has been measured in carbon foils [16], in the energy range of $\mathrm{MeV}$ per atom.

A theoretical treatment of the electronic stopping power of small clusters was first provided within the dielectric formalism [19]. In Ref. [20] the energy loss of slow hydrogen and water clusters was analyzed for an electron gas at finite temperature. The effects of the cluster structure and velocity on the stopping power were also evaluated [21,22] for swift and large hydrogen clusters in carbon and aluminum targets. A Monte Carlo code [23] was developed to treat the different processes involved in the interaction of hydrogen clusters, with energies in the keV/amu range, in thin carbon foils. Other recent developments include energy-loss studies of fullerenes in a Lindhard gas [24], and the cluster-size dependence of the stopping power for hydrogen clusters in silicon [25]. Besides, the analysis of collective effects in the energy loss of large clusters in plasmas [26-28] or solids [29] has also received recent attention due to the possibility of obtaining higher densities of deposited energy.

Our aim in this work is to evaluate how the stopping power of large molecular-hydrogen clusters depends on the cluster size and velocity. We also discuss the role played by the target nature on the above mentioned study. In particular, 
we analyze the case of aluminum and amorphous carbon, because they are commonly used as targets, and also because they have rather different dielectric properties.

The paper is organized as follows. Section II introduces the dielectric formalism used to obtain the cluster energy loss, and the model adopted to describe the dielectric properties of the target. In Sec. III we discuss the dependence of the cluster stopping power with the cluster size and velocity; the different results obtained for aluminum and amorphous carbon targets will be also analyzed. Finally, the conclusions are presented in Sec. IV.

\section{DIELECTRIC DESCRIPTION OF CLUSTER ENERGY LOSS}

\section{A. Stopping power}

When swift clusters impinge on a solid their binding electrons are stripped off after traversing the first atomic layers [2], forming then a cluster of atomic ions. These ions interact with the target and also among them (under the influence of Coulomb forces). However, since two time-scale regimes can be distinguished in these interactions (the electronic time-scale, $\sim 10^{-17}-10^{-16} \mathrm{~s}$, and the Coulomb explosion time-scale, $\sim 10^{-15}-10^{-14} \mathrm{~s}$ ), it is possible to evaluate separately both processes.

It should be noted that swift clusters inside solids are exploding projectiles in which each ion suffers changeexchange and multiple scattering interactions. Then, equilibrium times needed for the loss of the projectile electrons and for the proton charge equilibrium may be in some cases not negliglible when compared with Coulomb explosion times. However, the complexity of the whole problem makes a detailed study unpractical, therefore we will not consider these effects in our work.

In the context of swift cluster-beams bombarding a solid, when the cluster velocity $v$ is larger than Fermi velocity, $v_{F}$, it loses energy due mainly to the interaction with the target electrons. Then, in what follows we will consider only the electronic stopping power of swift clusters in solids, neglecting the contribution of nuclear stopping power.

The correlated motion of the cluster partners produces the so-called vicinage effects [2], which, up to first order perturbation theory, are satisfactorily evaluated within the dielectric formalism of the stopping power [19]. Within this framework, each cluster constituent is treated as a pointlike charge, and the target is modeled as an isotropic and homogeneous electron gas, whose response to an external perturbation is characterized completely by its dielectric function $\epsilon(k, \omega)$, where $k$ and $\omega$ are, respectively, the momentum and the energy transferred to electronic excitations in the medium. We will use atomic units throughout this paper.

When a charge penetrates into the solid it induces an electric field, which acts back on the projectile and causes it to lose kinetic energy. If we consider a cluster with velocity $\vec{v}$, composed by $N$ ions with relative positions $\vec{r}_{i j}$, then a general expression for the cluster stopping power, $S_{\mathrm{cl}}$, is given by [19]

$$
S_{\mathrm{cl}}=S_{p}\left[\sum_{i=1}^{N} Z_{i}^{* 2}+\sum_{i=1, i \neq j}^{N} Z_{i}^{*} Z_{j}^{*} I\left(r_{i j}, \theta_{i j}\right)\right],
$$

where $Z_{i}^{*}$ is the effective charge of each individual particle in the cluster. This effective charge is an average value that reflects the balance between the ionic electron-capture and loss processes. We assume that the effective charges $Z_{i}^{*}$ are all equal and identical for atomic and cluster ions, i.e., $Z_{i}^{*}=Z^{*} . r_{i j}$ are the distances between ions $i$ and $j$ in the cluster and $\theta_{i j}$ are the angles made by each relative vector position $\vec{r}_{i j}$ and the velocity $\vec{v}$ of the cluster. The stopping power of a single proton, $S_{p}$, is expressed as

$$
S_{p}=\frac{1}{2 \pi^{2} v} \int d^{3} k \frac{\vec{k} \cdot \vec{v}}{k^{2}} \operatorname{Im}\left[\frac{-1}{\epsilon(k, \omega=\vec{k} \cdot \vec{v})}\right]
$$

The vicinage effects are explicitly taken into account through the interference function $I(r, \theta)$, which can be written as [20]

$$
I(r, \theta)=\frac{1}{2 \pi^{2} v S_{p}} \int d^{3} k \frac{\vec{k} \cdot \vec{v}}{k^{2}} \operatorname{Im}\left[\frac{-1}{\epsilon(k, \omega=\vec{k} \cdot \vec{v})}\right] \cos (\vec{k} \cdot \vec{r}) .
$$

A random distribution of the particles in the cluster is suitable for large clusters [22]. After doing an angular average in Eq. (3), and introducing the cluster pair distribution function $g_{\mathrm{cl}}(r)$, which accounts for the correlated positions of the particles in the cluster [20,22], we obtain the following expression for the cluster stopping power:

$$
\begin{aligned}
S_{\mathrm{cl}}= & N Z^{* 2}\left\{S_{p}+\frac{2 \bar{n}}{\pi v^{2}} \int_{0}^{\infty} \frac{d k}{k} \frac{\sin k r}{k r} \int d^{3} r g_{\mathrm{cl}}(r)\right. \\
& \left.\times \int_{0}^{k v} d \omega \omega \operatorname{Im}\left[\frac{-1}{\epsilon(k, \omega)}\right]\right\}
\end{aligned}
$$

with $\bar{n}$ being the average nuclear density of the cluster; in our case we take $\bar{n}=6.27 \times 10^{-3}$ a.u. for hydrogenic clusters, according to Ref. [30].

\section{B. Cluster geometry}

The cluster geometry is described through the pair distribution function $g_{\mathrm{cl}}(r)$, which gives the probability that two particles in the cluster are at a relative distance $r$. This distribution satisfies the normalization condition $N=1+\bar{n} \int d^{3} r g_{\mathrm{cl}}(r)$, where the integration extends to the volume of the cluster. Since we are interested in large clusters of molecular hydrogen, $\left(\mathrm{H}_{2}\right)_{n}$, we separate $g_{\mathrm{cl}}(r)$ in two contributions: $g_{\text {cl }}(r)=g_{\text {intra }}(r)+g_{\text {inter }}(r)$. The intramolecular term, $g_{\text {intra }}(r)$, describes the almost fixed separation of the protons in each $\mathrm{H}_{2}$ molecule, while the intermolecular term, $g_{\text {inter }}(r)$, provides the spatial distribution of the rest of molecules in the cluster.

The structure of the $\mathrm{H}_{2}$ molecule was represented by a $\delta$ distribution, $g_{\text {intra }}(r)=\left(4 \pi r_{\text {mol }}^{2} \bar{n}\right)^{-1} \delta\left(r-r_{\text {mol }}\right)$, where $r_{\mathrm{mol}}=1.40$ a.u. is the corresponding internuclear distance [30]. The intermolecular pair correlation function $g_{\text {inter }}(r)$ was modeled by a random distribution of intermolecular distances in a spherical cluster with an exclusion volume around each molecule, due to the repulsive interaction between $\mathrm{H}_{2}$ molecules at close distances; this exclusion distance is taken $r_{\mathrm{ex}}=3.59$ a.u. [30]. Finally, the finite spherical geometry of 
the cluster was also incorporated in $g_{\mathrm{cl}}(r)$, according to the procedure presented in Ref. [22].

We define the reduced stopping power as the stopping power per particle, normalized to the square of the effective charge, namely, $S_{\mathrm{cl}} /\left(N Z^{* 2}\right)$. It can be separated into contributions due to each proton not being part of the cluster, $S_{p}$, the interference effects produced by the protons of the same molecule, $S_{\text {intra }}$, and the interference generated by protons from different molecules, $S_{\text {inter }}$. Then we can write

$$
\frac{S_{\mathrm{cl}}}{N Z^{* 2}}=S_{p}+S_{\text {intra }}+S_{\text {inter }} .
$$

\section{Dielectric description of the target}

The cluster energy loss [see Eq. (4)] depends on the target properties through the energy loss function (ELF, hereafter), $\operatorname{Im}[-1 / \epsilon(k, \omega)]$, which should be specified for each of the targets considered in this work: aluminum and amorphous carbon.

We model the ELF using the dielectric function introduced by Mermin [31], $\epsilon_{M}$, which is a generalization of the Lindhard dielectric function [32] in the relaxation time approximation. With this formalism, the finite lifetime for the plasmon oscillations and their decay into individual excitations are properly taken into account.

The procedure we have used to model the ELF has been described in detail elsewhere [33,34], where we have shown that it gives satisfactory estimations of proton stopping powers [33], as well as an accurate description of the $k$ and $\omega$ dependence of the ELF [34]. In brief, we construct the ELF by fitting the experimental ELF, usually available at zero wave number, by means of a sum of Mermin-type ELF's. The number of terms depends roughly on the number of peaks in the ELF:

$$
\operatorname{Im}\left[\frac{-1}{\epsilon(k, \omega)}\right]=\sum_{i} A_{i} \operatorname{Im}\left[\frac{-1}{\epsilon_{M}\left(\omega_{p l, i}, \gamma_{i}, k, \omega\right)}\right] .
$$

The parameters $\omega_{p l, i}$, and $\gamma_{i}$ are related to the energy position and width of the peaks in the ELF, respectively, and the coefficients $A_{i}$ are chosen in such a manner that the $f$-sum rule

$$
N_{\text {eff }}(\omega)=\frac{1}{2 \pi^{2} \rho} \int_{0}^{\omega} d \omega^{\prime} \omega^{\prime} \operatorname{Im}\left[\frac{-1}{\epsilon\left(k, \omega^{\prime}\right)}\right]
$$

will be satisfied (this gives the effective number of electrons per atom that participate in the excitations up to energy $\omega$ ). In the above expression, $\rho$ is the target atomic density.

Using the experimental ELF at $k=0$ for aluminum [35] and for amorphous carbon [36] we obtain the set of $A_{i}$, $\omega_{p l, i}$, and $\gamma_{i}$ values reported in Table I. In Fig. 1 we have depicted the experimental and fitted ELF's of aluminum [Fig. 1(a)] and amorphous carbon [Fig. 1(b)]. The aluminum energy loss spectrum presents a very well defined peak at the energy $\omega_{p l} \approx 0.55$ a.u. in the large wavelength limit, as can be seen in Fig. 1(a). The ELF of amorphous carbon clearly shows two peaks, which describe the collective excitations of $\pi$ and $(\pi+\sigma)$ electrons in carbon [36,37]; experimental evi-
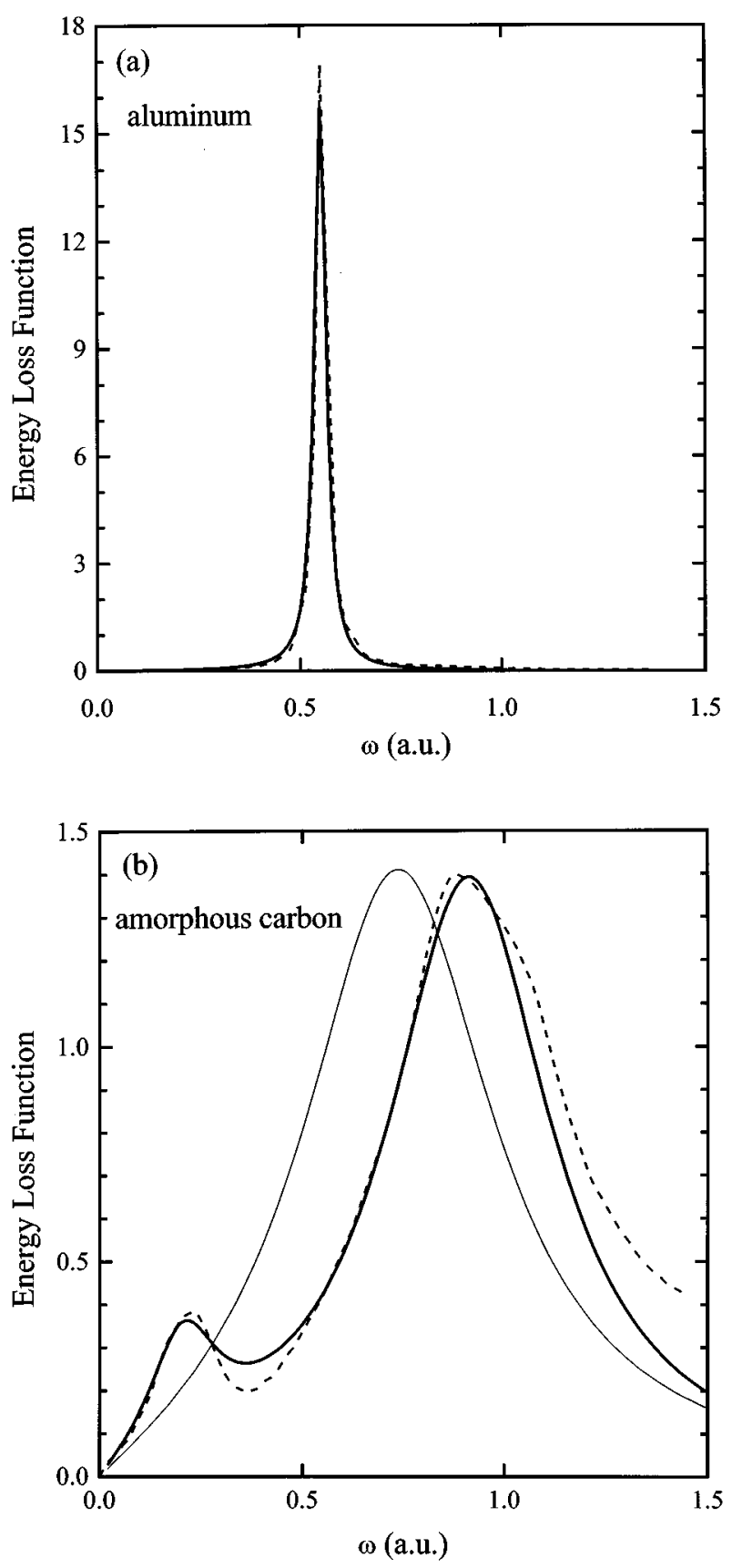

FIG. 1. (a) Energy loss function of aluminum. Dashed line, experimental data [35]; solid line, fitted ELF using Eq. (6). (b) Energy loss function of amorphous carbon. Dashed line, experimental data [36]; thick solid line, fitted ELF using Eq. (6) using two Mermintype ELF's; thin solid line, fitted ELF using a single Mermin ELF with the plasmon energy obtained from the amorphous carbon density.

dence [38] also indicates the presence of large damping of the plasma oscillations in carbon.

In some previous studies $[3,21,39]$ the carbon ELF has been described with a single plasmon energy $\omega_{p l}=0.792$ a.u., obtained according to the relation $\omega_{p l}^{2}=4 \pi \mathcal{N}$, where $\mathcal{N}$ is the free electron density of carbon, and with a damping $\gamma=0.58$ a.u. To illustrate the need for a more accurate target 
TABLE I. Parameters used to fit the ELF of aluminum and amorphous carbon through Eq. (6).

\begin{tabular}{|c|c|c|c|c|c|c|}
\hline \multirow[b]{2}{*}{$i$} & \multicolumn{3}{|c|}{ Aluminum } & \multicolumn{3}{|c|}{ Amorphous carbon } \\
\hline & $A_{i}$ & $\omega_{p l, i}$ (a.u.) & $\gamma_{i}$ (a.u.) & $A_{i}$ & $\omega_{p l, i}$ (a.u.) & $\gamma_{i}$ (a.u.) \\
\hline 1 & 1.1178 & 0.551 & 0.035 & 0.2369 & 0.23 & 0.21 \\
\hline 2 & & & & 0.7083 & 0.945 & 0.49 \\
\hline
\end{tabular}

description, in the next section we will compare the predictions of the cluster stopping powers obtained using the single plasmon ELF or the sum of Mermin-type ELF's.

\section{RESULTS AND DISCUSSION}

We will now consider the dependence, on cluster size and velocity, of the stopping power of $\left(\mathrm{H}_{2}\right)_{n}$ clusters on aluminum and amorphous carbon. We compare the prediction for these two materials that present different dielectric responses: the aluminum ELF, characterized by one very-welldefined plasmon with small damping [35], and the amorphous carbon EFL, composed essentially of two plasmon excitations with large damping [36].

\section{A. Aluminum target}

First, we study the stopping power of $\left(\mathrm{H}_{2}\right)_{n}$ clusters impinging on aluminum targets. In Fig. 2 we show the results of $S_{p}, S_{\text {intra }}$, and $S_{\text {inter }}$ versus cluster velocity $v$; the behavior of $S_{p}$ satisfactorily agrees with the compiled experimental data $[40-43]$.

At low velocities, $S_{p}$ follows a linear dependence with $v$, reaches a maximum at $v \sim 1.5$ a.u., and decreases for large velocities. The behavior of $S_{\text {intra }}$ with velocity is similar to

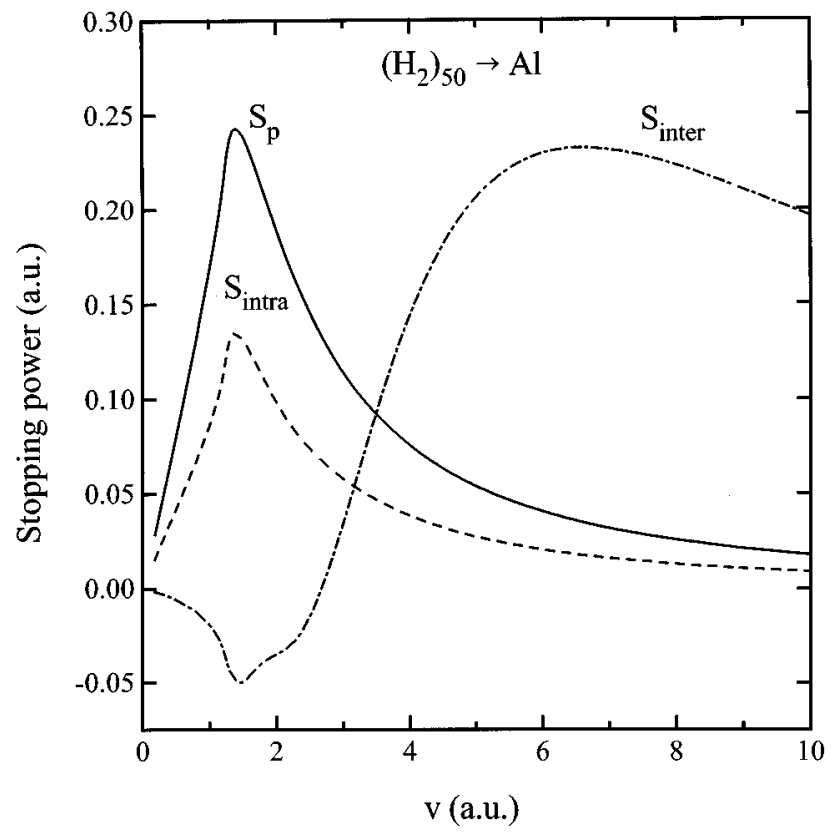

FIG. 2. Reduced stopping powers, $S_{p}, S_{\text {intra }}$, and $S_{\text {inter }}$, as a function of the cluster velocity. We present results for a cluster with 100 particles incident on aluminum.
$S_{p}$ but its values are roughly half. The velocity dependence of $S_{\text {inter }}$ for a cluster composed of 100 particles predicts a small and negative value for low velocities, but then increases and finally dominates the cluster energy loss at large velocities. A similar result was obtained in Ref. [22] but using a $\delta$ function to describe the ELF of aluminum.

To analyze the size dependence of the cluster stopping power we only study the term $S_{\text {inter }}$, since $S_{p}$ and $S_{\text {intra }}$ are independent of the number of atoms in the cluster. In Fig. 3 we present the intermolecular stopping power $S_{\text {inter }}$ as a function of cluster size, i.e., the number of particles in the cluster, for various velocities. We emphasize that for cluster velocities $v \geqslant 2$ a.u., we obtain a well-defined maximum in $S_{\text {inter }}$. This means that for each velocity there is a cluster, to be called "resonant cluster,' for which the energy loss per particle is maximum. This resonant effect takes place when the interference among all the cluster components is constructive and the collective effect is maximum. We note also that the resonant cluster is achieved for intermediate cluster sizes and not for the biggest clusters. A similar outcome has been obtained for the stopping of hydrogen clusters in silicon [25] as well as in plasma targets [28].

The size of the resonant cluster and the corresponding maximum value of $S_{\text {inter }}$ increase with the cluster velocity. We characterize the resonant cluster configuration by the ra-

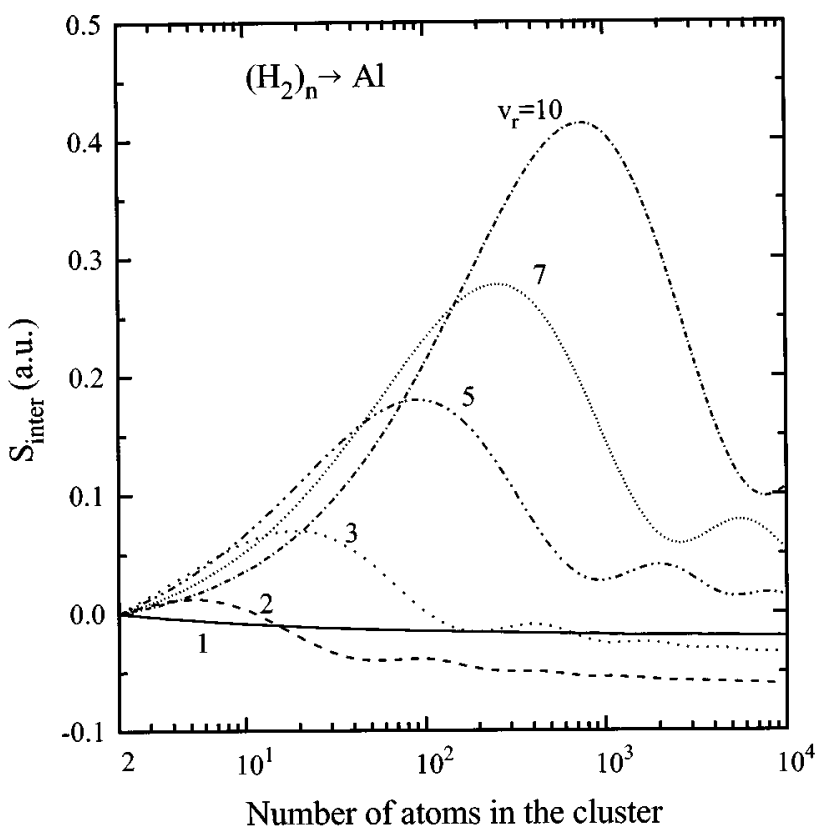

FIG. 3. Reduced intermolecular stoppping power, $S_{\text {inter }}$, for different cluster velocities $v_{r}\left(v_{r}=v / v_{F}\right)$, plotted as a function of the number of particles in the cluster. The target is aluminum, and $v_{F}=0.894$ a.u. 


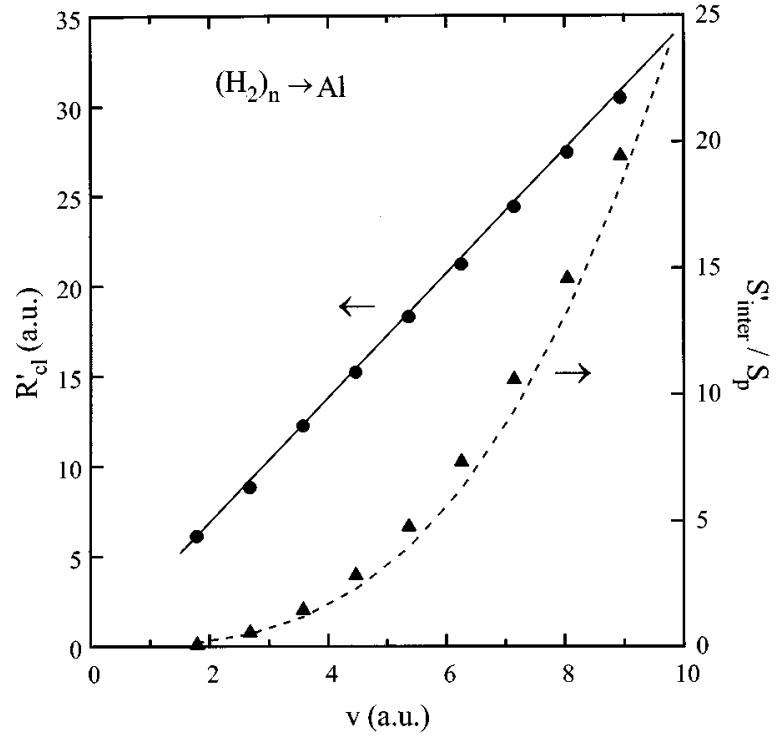

FIG. 4. The left axis represents the radius $R_{\mathrm{cl}}^{\prime}$ for the resonant cluster (at which a maximum in the $S_{\text {inter }}$ appears) as a function of the cluster velocity. Dots, cluster radius for the maximum $S_{\text {inter }}$, derived from Fig. 3; line, scale rule given by Eq. (8). The right axis shows the maximum value of the intermolecular stopping power $S_{\text {inter }}^{\prime}$ (normalized to the proton stopping power, $S_{p}$ ) as a function of the velocity. Triangles, values derived from Fig. 3; dashed line, scale rule given by Eq. (9).

dius $R_{\mathrm{cl}}^{\prime}$ and the maximum value of the intermolecular stopping power $S_{\text {inter }}^{\prime}$ In Fig. 4 we present the dependence of $R_{\mathrm{cl}}^{\prime}$ and $S_{\text {inter }}^{\prime}$ with the cluster velocity, respectively. As we can see, the resonant cluster radius follows a linear dependence with the cluster velocity. The plasmon pole approximation to describe the target predicts that the maximum interference will be when the plasmon wavelength is similar to the cluster radius, that is,

$$
R_{\mathrm{cl}}^{\prime} \cong \frac{2 v}{\omega_{\mathrm{pl}}} .
$$

This scale rule, shown as a solid line in Fig. 4, agrees very well with the theoretical data obtained by our model, represented by dots in Fig. 4. This means that, as discussed in Ref. [22], the main contribution to the collective effects in the cluster stopping power is due to the interferences between target plasmon oscillations generated by the several particles that compose the cluster.

The maximum value of $S_{\text {inter }}$ divided by the proton stopping power $S_{p}$ is shown also in Fig. 4 as a function of the cluster velocity. The theoretical data follow approximately the relation

$$
\frac{S_{\text {inter }}^{\prime}}{S_{p}} \cong 0.8 n\left(\frac{v}{\omega_{\mathrm{pl}}}\right)^{3}
$$

and since at high velocities $S_{p} \propto v^{-2}$, then $S_{\text {inter }}^{\prime}$ goes linearly with the cluster velocity. Therefore, the collective effects become relatively more important with increasing velocities.

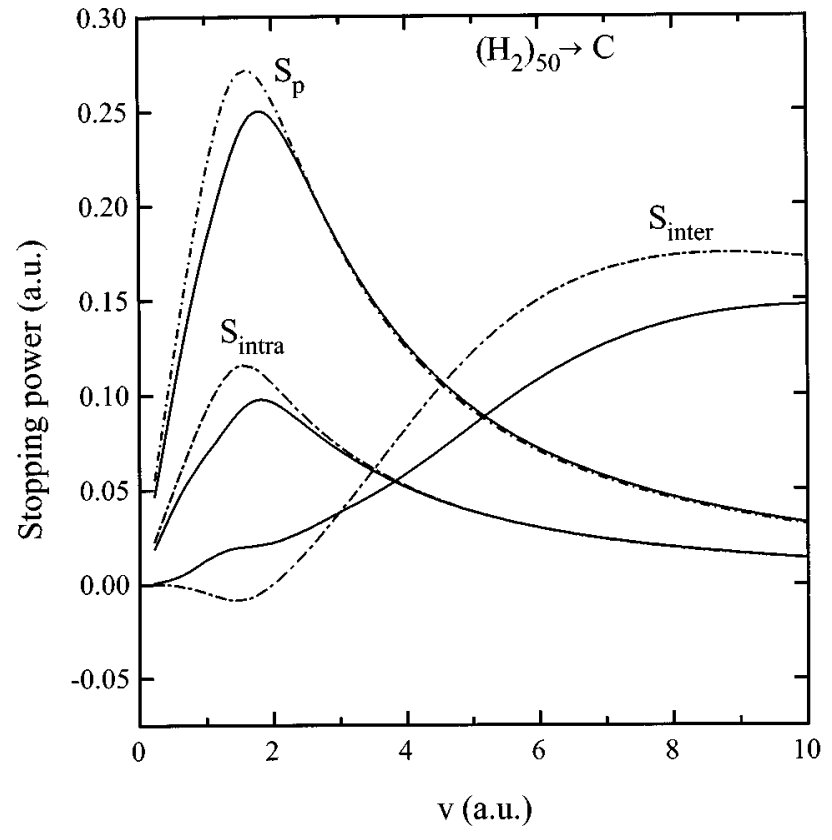

FIG. 5. Velocity dependence of the reduced cluster stopping power: $S_{p}, S_{\text {intra }}$, and $S_{\text {inter }}$. We present data for a cluster with 100 particles, incident on amorphous carbon, whose ELF is modeled by a sum of two Mermin ELF's (solid line) or a single Mermin ELF (dot-dashed line).

Similar results were obtained for the energy loss of hydrogen clusters in silicon targets [25] and in plasmas [28].

\section{B. Amorphous carbon target}

Now we analyze the stopping power of $\left(\mathrm{H}_{2}\right)_{n}$ clusters in amorphous carbon, as a function of the velocity and cluster size. The ELF for amorphous carbon was modeled here as a sum of two Mermin ELF's [see Eq. (6)], and also for comparison, using only one Mermin ELF. The two-component model for the ELF provides a more realistic representation of the two resonances observed in the optical spectra of amorphous carbon [36,37] [see Fig. 1(b)].

We consider a cluster of hydrogen, composed by 100 particles, bombarding an amorphous carbon target. In Fig. 5 we show the velocity dependence of the relevant terms in the stopping power [see Eq. (5)]: $S_{p}, S_{\text {intra }}$, and $S_{\text {inter }}$. The solid lines correspond to the results obtained with the twoMermin-ELF's model, while the dot-dashed lines represent the results derived from the simpler (but more inaccurate, as we will see below) single-Mermin-ELF model. At low velocities, $S_{p}$ goes linearly with $v$, reaches a maximum at $v \sim 1.5$ a.u., and then decreases with velocity. In Ref. [33] it was shown that using a sum of two Mermin ELF's the $S_{p}$ of amorphous carbon agrees fairly well with the experimental data. The intramolecular stopping term, $S_{\text {intra }}$, presents similar trends to $S_{p}$, but its values are about $45 \%$ of those of $S_{p}$ in all the range of velocities. The behaviors of $S_{p}$ and $S_{\text {intra }}$ are similar for both dielectric descriptions of carbon. The model based in a single Mermin ELF gives more stopping than the model using two Mermin ELF's; this is so because the $\omega_{p l}$ value used in the single plasmon model is smaller than the high-plasmon peak in the experimental ELF.

However, the results obtained for $S_{\text {inter }}$ are very different depending of the model used to describe the target. At low 
cluster velocities we obtain negative values of $S_{\text {inter }}$ when we use the one-component ELF; this is due to destructive interferences in the stopping power. We have found similar results in calculations for aluminum and silicon targets (whose ELF's are well described with a single plasmon peak) and it was observed that these negative values become bigger the narrower is the plasmon resonance. However, when we include the two plasma resonances in the ELF the destructive interferences disappear, and we obtain positive values of $S_{\text {inter }}$ in the whole velocity range. In this case, $S_{\text {inter }}$ grows with velocity until it seems to saturate for $v>8$ a.u., while in the case of a single Mermin ELF we obtain bigger values of $S_{\text {inter }}$. We find that this apparent saturation corresponds to a smooth maximum, but one should go to still higher velocities to find the subsequent decline.

By comparing the values of the reduced intermolecular and intramolecular stopping terms with the proton stopping power, we find that the intermolecular collective effect is dominant when the cluster velocity satisfies $v_{r} \geqslant 5$, where $v_{r}=v / v_{F}$, and $v_{F}=1.138$ a.u. For instance, when $v_{r}=5$ we obtain $S_{p}=0.077$ a.u., $S_{\text {intra }}=0.032$ a.u. and $S_{\text {inter }}=0.101$ a.u. for a cluster made of 100 particles.

Also if we compare the values of $S_{\text {inter }}$ for aluminum and amorphous carbon, the conclusion is that the vicinage effects decrease for those materials that present several (and wider) plasmon resonances in the ELF. These effects will be larger for materials with only one resonance frequency, being more sizeable the longer the mean life of the collective excitations produced in the target.

Now, we evaluate the dependence with the cluster size of the vicinage effect in the stopping power of hydrogen clusters impinging in amorphous carbon. We center our study in the intermolecular stopping power $S_{\text {inter }}$. The reduced intermolecular stopping power $S_{\text {inter }}$, as a function of the number of atoms in the cluster, is shown in Fig. 6(a) when the target is described by a sum of two Mermin ELF's, and in Fig. 6(b) when a single Mermin ELF was used. We present results for various cluster velocities, $1 \leqslant v_{r} \leqslant 6$.

From Fig. 6(a), we observe that, when $v_{\mathrm{r}}=1, S_{\text {inter }}$ as a function of the number of particles in the cluster shows a saturation (or a very flat maximum), starting approximately from ten particles. Similar trends were reported experimentally by Ray et al. [15] for the energy loss of hydrogen clusters in carbon foils at $v \sim 1$ a.u.; these experiments found a saturation in the energy loss for small clusters. Unfortunately, at present there are no stopping power experiments for high velocities and large cluster sizes. This saturation effect at low velocities could be due to the fact that the projectile velocity is not high enough to excite plasmons, which would produce a coherent interference or resonant behavior, as we will discuss below. A different behavior arises for cluster velocities $v_{r}>2$. By comparison with the simplest case of aluminum, and following from Eq. (8), we may expect the appearance of two maxima in the stopping power, associated each with the low- and high-energy plasmons in the amorphous carbon target. Actually, we observe that as a result of this double-plasmon feature (included in the amorphous carbon ELF), a plateau is formed for intermediate cluster sizes $(N \sim 100)$. We also find that the position of the second maximum shifts to larger cluster sizes for $v_{r}>4$, falling beyond the range covered in this figure.
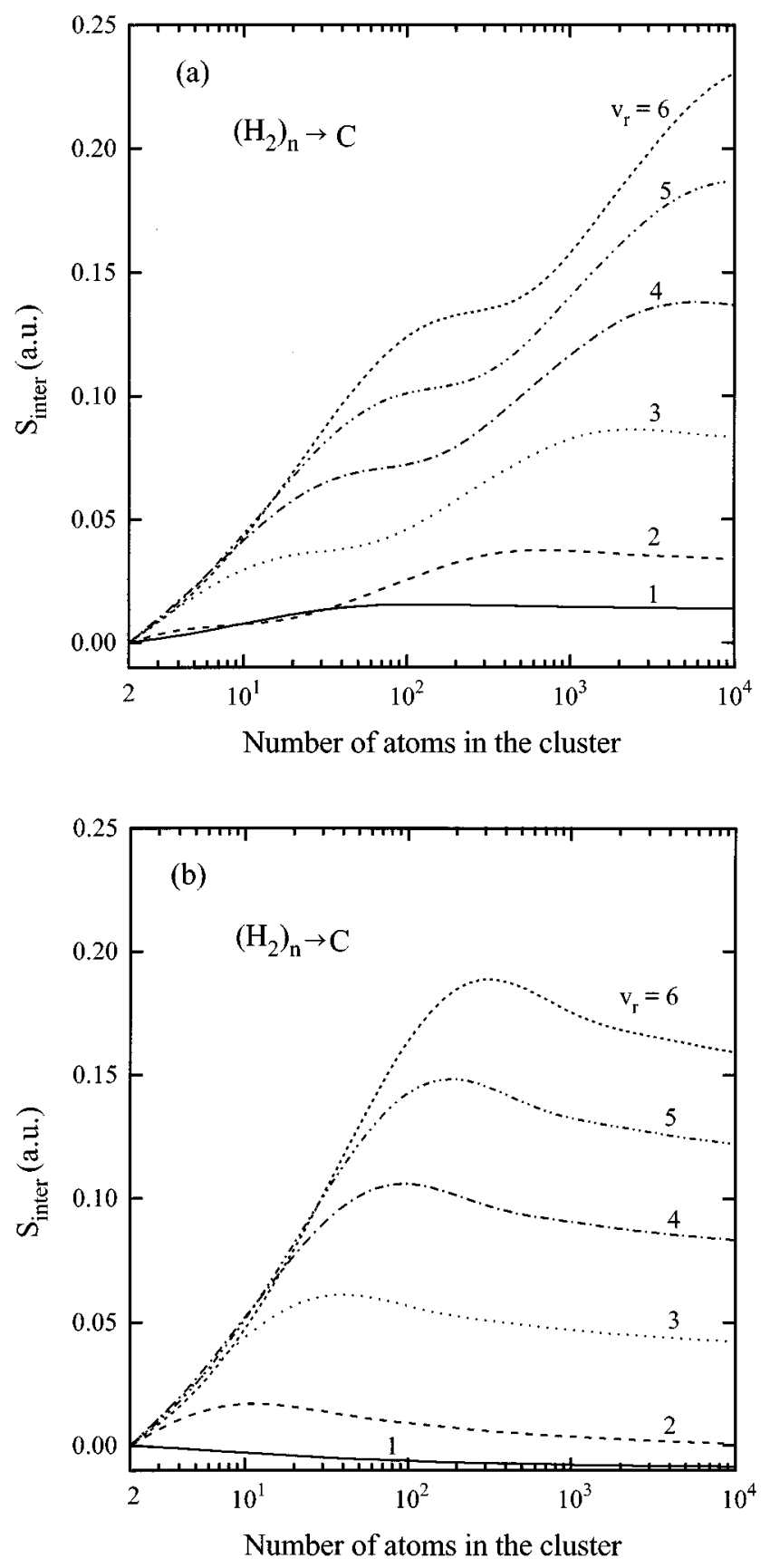

FIG. 6. $S_{\text {inter }}$ versus number of particles in the cluster when the ELF of the amorphous carbon target is described: (a) as sum of two Mermin ELF's, and (b) by a single Mermin ELF. Different curves are displayed for different cluster velocities, $v_{r}=v / v_{F}$, with $v_{F}=1.138$ a.u.

An attempt to compare our predictions with the experimental results from Ref. [15] is not possible, because these experiments were done with small clusters, and our model (spherical and homogeneous clusters) works better for large than for small clusters; moreover, the structure of the projectiles used in the experiments (a core $\mathrm{H}_{3}^{+}$ion surrounded by neutral $\mathrm{H}_{2}$ molecules) differs from the structure we have considered.

When using the single-Mermin-ELF model to evaluate the stopping power, Fig. 6(b), we observe that $S_{\text {inter }}$ shows a 


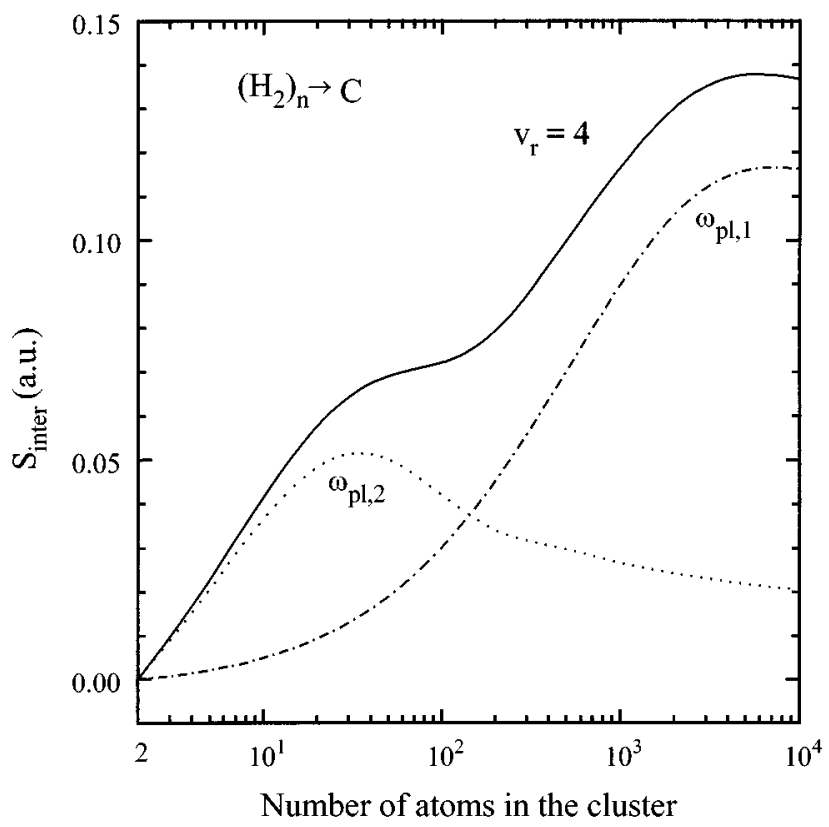

FIG. 7. Contribution of each plasmon peak in the ELF of amorphous carbon to the intermolecular stopping power, $S_{\text {inter }}$, as a function of the number of particles in the cluster. The solid curve, $S_{\text {inter }}$, is the sum of the contribution due to the low-plasmon peak (dot-dashed curve) and the high-plasmon peak (dotted curve). The velocity of the cluster relative to the Fermi velocity of amorphous carbon is $v_{r}=4$. The amorphous carbon target was modeled by a sum of two Mermin ELF's.

soft maximum as a function of the number of particles in the cluster, which substantially differs from the previously discussed behavior. Although this maximum is not so pronounced as in the case of aluminum (Fig. 3), it becomes more evident at higher velocities.

In order to understand these results we present in Fig. 7 the contributions to the value of $S_{\text {inter }}$ arising from each of the two plasma resonances $\left(\omega_{\mathrm{pl}, 1}=0.23\right.$ a.u. and $\omega_{\mathrm{pl}, 2}=0.945$ a.u.) in the ELF of amorphous carbon [cf. Fig. 1(b)] for the case of $v_{r}=4$. The dot-dashed curve in Fig. 6(a) is the same that the solid curve in Fig. 7, where the dotted line represents the contribution of the high-plasmon $\left(\omega_{\mathrm{pl}, 2}\right)$ and the dot-dashed line the one due to the low-plasmon $\left(\omega_{\mathrm{pl}, 1}\right)$. We can observe that the intermolecular stopping term, $S_{\text {inter }}$, is the superposition of two contributions, due to each of these resonances. In addition, we find that the first peak in $S_{\text {inter }}$ [appearing as a shoulder at $N \sim 30-100$ in Fig. 6(a) for the case $\left.v_{r}=4\right]$ is due to the high plasma resonance $\left(\omega_{\mathrm{pl}, 2}=0.945\right.$ a.u. $)$ in the carbon spectrum of Fig. 1(b), whereas the largest enhancement effect [second peak, at $N \sim 5000$, in Fig. 6(a)] is produced by the smaller low-energy plasma resonance $\left(\omega_{\mathrm{pl}, 1}=0.23\right.$ a.u.) in Fig. 1(b). This seems to support the idea that the low plasmon peak in the ELF has an important contribution to the stopping power and, in fact, it becomes dominant for high velocities and large clusters.

Therefore, these results show both the influence of the low energy resonance on the long-range behavior of the dynamical interactions for large clusters of ions, and the necessity of more accurate EFL models taking into account in a more realistic way the structure of the energy loss peaks determined experimentally.

\section{CONCLUSIONS}

The analysis of the energy loss of large clusters of ions in matter, based on the standard dielectric function formalism, but using a more realistic description of the target properties, predicts new and interesting features of the dynamical interactions that directly affect the energy dissipated in the medium.

We propose an approach in which the target electronic properties, determined from the experimental analysis of the energy-loss functions of aluminum and amorphous carbon targets, are represented by a combination of Mermin-type dielectric functions. In this way, the perturbations produced by the cluster of particles are taken into account in a more accurate way than in previous electron-gas descriptions.

In the case of aluminum, where a well-defined single plasmon resonance is observed experimentally [35], our results predict an important resonant behavior in the cluster stopping power, which occurs when the size of the cluster is such that most of its particles interfere in a coherent way with the collective perturbation of the medium. The radius of the resonant cluster, for which a maximum stopping enhancement is obtained, is proportional to the velocity [Eq. (8)], whereas the maximum intermolecular stopping power value, divided by the proton stopping power, is proportional to the third power of the velocity [Eq. (9)].

A different behavior is obtained in the case of amorphous carbon, because of the double-plasmon characteristics observed in the primary energy-loss spectra [36]. In this case, in the range of high velocities $\left(v_{r}>3\right)$ the contributions from the two broad resonances partially overlap, producing a plateau for intermediate cluster sizes, and a wide maximum for very large clusters. Moreover, for intermediate velocities $\left(v_{r} \sim 1-2\right)$ we find an approximate saturation in the cluster energy loss when the number of particles in the cluster is increased. This behavior is qualitatively in agreement with recent experimental observations of saturation effects in the energy loss of hydrogen clusters in carbon foils [15].

We emphasize here the importance of using an accurate representation of the energy loss function of each material in order to provide a good description of the interference effects, in particular in the case of swift ion clusters where these effects extend over a long range of distances and become sensitive to the structure of the dielectric function.

We think that a realistic study of multiatomic cluster collisions and propagation inside a solid should incorporate many more processes than those discussed in this paper: cluster spread, charge exchange, multiple scattering, and electronic excitations. However, at the present time, some of these effects have not yet been analyzed enough.

\section{ACKNOWLEDGMENTS}

Partial support was provided by the Spanish Dirección General de Investigación Científica y Técnica, through projects PB92-0341 and PB93-1125. N.R.A. thanks the Conselleria d'Educació i Ciència de la Generalitat Valenciana for financial support under the program PROPIO. 
[1] Proceedings of the Conference on Polyatomic Ion Impact on Solids and Related Phenomena, Saint-Malo, 1993, edited by H. H. Andersen and L. E. Rehn [Nucl. Instrum. Methods B 88, 1 (1994)].

[2] W. Brandt, A. Ratkowsky, and R. H. Ritchie, Phys. Rev. Lett. 33, 1325 (1974).

[3] M. F. Steuer and R. H. Ritchie, Nucl. Instrum. Methods B 33, 170 (1988); 40/41, 372 (1989).

[4] Y. Wang and T. Ma, Phys. Lett. A 178, 209 (1993).

[5] A. Bret, Nucl. Instrum. Methods B 88, 107 (1994).

[6] J. Jensen, H. H. Mikkelsen, and P. Sigmund, Nucl. Instrum. Methods B 88, 191 (1994).

[7] M. M. Jakas and N. E. Capuj, Phys. Rev. A 52, 439 (1995).

[8] T. Kaneko, Phys. Rev. A 51, 535 (1995).

[9] D. S. Gemmell, A. Faibis, E. P. Kanter, Z. Vager, and B. J. Zabransky, Vacuum 39, 355 (1989).

[10] Y. Susuki, K. Kimura, M. Mannami, N. Sakamoto, H. Ogawa, I. Katayama, T. Noro, and H. Ikegami, Phys. Rev. A 50, 3533 (1994).

[11] M. Fritz, K. Kimura, Y. Susuki, and M. Mannami, Phys. Rev. A 50, 2405 (1994).

[12] B. Mazuy, A. Belkacem, M. Chevallier, M. J. Gaillard, J. C. Poizat, and J. Remillieux, Nucl. Instrum. Methods B 28, 497 (1987)

[13] B. Mazuy, A. Belkacem, M. Chevallier, M. J. Gaillard, J. C. Poizat, and J. Remillieux, Nucl. Instrum. Meth. B 33, 105 (1988).

[14] M. Farizon, A. Clouvas, N. V. Castro Faria, B. FarizonMazuy, M.-J. Gaillard, E. Gerlic, A. Denis, J. Desesquelles, and Y. Ouerdane, Phys. Rev. A 43, 121 (1991).

[15] E. Ray, R. Kirsch, H. H. Mikkelsen, J. C. Poizat, and J. Remillieux, Nucl. Instrum. Methods B 69, 133 (1992).

[16] K. Baudin, A. Brunelle, M. Chabot, S. Della-Negra, J. Depauw, D. Gardès, P. Håkansson, Y. Le Beyec, A. Billebaud, M. Fallavier, J. Remillieux, J. C. Poizat, and J. P. Thomas, Nucl. Instrum. Methods B 94, 341 (1994).

[17] J. C. Eckardt, G. Lantschner, N. R. Arista, and R. A. Baragiola, J. Phys. C 11, L851 (1978).

[18] R. Levi-Setti, K. Lam, and T. R. Fox, Nucl. Instrum. Methods 194, 281 (1982).

[19] N. R. Arista, Phys. Rev. B 18, 1 (1978).

[20] N. R. Arista and A. Gras-Martí, J. Phys. Condens. Matter 3, 7931 (1991).
[21] I. Abril, M. Vicanek, A. Gras-Martí, and N. R. Arista, Nucl. Instrum. Methods B 67, 56 (1992).

[22] M. Vicanek, I. Abril, N. R. Arista, and A. Gras-Martí, Phys. Rev. A 46, 5745 (1992).

[23] M. Farizon, N. V. de Castro Faria, B. Farizon-Mazuy, and M. J. Gaillard, Phys. Rev. A 145, 179 (1992).

[24] E. Nardi and Z. Zinamon, Phys. Rev. A 51, R3407 (1995).

[25] F. J. Pérez-Pérez, I. Abril, N. R. Arista, and R. Garcia-Molina, Nucl. Instrum. Methods B 115, 18 (1996).

[26] E. Nardi, Z. Zinamon, and D. Ben-Hamu, Nuovo Cimento 106, 1839 (1993).

[27] C. Deutsch and N. A. Tahir, Nuovo Cimento 106, 1811 (1993).

[28] E. Bringa and N. R. Arista, Phys. Rev. E 52, 3010 (1995).

[29] C. Deutsch and N. A. Tahir, Laser Part. Beams 13, 211 (1995).

[30] I. F. Silvera, Rev. Mod. Phys. 52, 393 (1980).

[31] N. D. Mermin, Phys. Rev. B 1, 2362 (1970).

[32] J. Lindhard, Mat. Fys. Medd. Dan. Vid. Selsk. 28, No. 8 (1954).

[33] I. Abril, R. Garcia-Molina, and N. R. Arista, Nucl. Instrum. Methods B 90, 72 (1994).

[34] D. J. Planes, R. Garcia-Molina, I. Abril, and N. R. Arista, J. Electron Spectrosc. Relat. Phenom. (to be published).

[35] H.-J. Hagemann, E. Gudat, and C. Kunz, J. Opt. Soc. Am. 65, 742 (1975); H.-J. Hagemann, E. Gudat, and C. Kunz, Deutsches Elektronen-Synchrotron Report No. DESY SR-74/7, Hamburg, 1974.

[36] J. Cazaux and D. Gramari, J. Phys. (Paris) 38, L133 (1977).

[37] R. Raether, Excitations of Plasmons and Interband Transitions by Electrons, Springer Tracts in Modern Physics Vol. 88 (Springer, Berlin, 1980).

[38] M. W. Williams and E. T. Arakawa, J. Appl. Phys. 43, 3460 (1972).

[39] J. C. Ashley and P. M. Echenique, Phys. Rev. B 16, 8701 (1987).

[40] H. H. Andersen and J. F. Ziegler, Hydrogen: Stopping Powers and Ranges in All Elements, Vol. 3 of The Stopping and Ranges of Ions in Matter (Pergamon, New York, 1977).

[41] J. F. Janni, At. Data Nucl. Data Tables 27, 341 (1982).

[42] J. F. Ziegler, J. P. Biersack, and U. Littmark, The Stopping and Range of Ions in Solids, Vol. 1 of The Stopping and Ranges of Ions in Matter (Pergamon, New York, 1985).

[43] International Commission on Radiation Units and Measurements Report No. 49, Bethesda, MD, 1993 (unpublished). 